Research Article

\title{
Research on the Fracture Grouting Mechanism and PFC Numerical Simulation in Loess
}

\author{
Zhenlong Zhang $\mathbb{D},{ }^{1}$ Zhushan Shao $\mathbb{D},{ }^{1}$ Xiaobo Fang, ${ }^{1}$ and Xijun Liang ${ }^{2}$ \\ ${ }^{1}$ School of Civil Engineering, Xi'an University of Architecture \& Technology, Xi'an 710055, China \\ ${ }^{2}$ China Railway First Group Co., Ltd., Xi'an 710054, China \\ Correspondence should be addressed to Zhenlong Zhang; 370331615@qq.com
}

Received 9 July 2017; Accepted 4 June 2018; Published 26 July 2018

Academic Editor: Aniello Riccio

Copyright (c) 2018 Zhenlong Zhang et al. This is an open access article distributed under the Creative Commons Attribution License, which permits unrestricted use, distribution, and reproduction in any medium, provided the original work is properly cited.

According to the model test of fracture grouting in loess, the grout changes with the pressure variation, which can be divided into three stages, foundation, expansion and fracturing, and diffusion, and ultimately splits into "Y" shaped grout fractures. Using $\mathrm{PFC}^{2 \mathrm{D}}$ particle flow software simulates the slurry diffusion process on loess, and the results of the simulation display the same "Y" shaped slurry vein with the experiment, which has a certain reference value for fracture grouting in loess.

\section{Introduction}

Fracture grouting, as an effective means of support and reinforcement for tunnel construction, is widely used in loess areas. The recent interest in fracture grouting is highlighted by a large number of symposia and scholar through theoretical researches [1-4], model tests [5-7], and numerical simulation $[8,9]$. The fracture grouting process is complex and hard to track, which involves multiple disciplines, including geotechnical mechanics, fluid mechanics, and material mechanics, and current researches are still far behind the engineering application [1].

The experimental study of fracture grouting is mainly focused on soft soil, and the research on the splitting, diffusion process, and spatial geometry of the fracture grouting in loess is very rare. The complexity of loess results in some degree of dispersion and distortion of model test results, which affects the accuracy of scientific research and engineering design. The numerical simulation test can complement the model test and is an important research method.

In this paper, the formation and development of grout fractures during fracture grouting are analyzed in depth through numerous model tests. $\mathrm{PFC}^{2 \mathrm{D}}$ particles flow analysis program is used to establish the loess mesoscopic numerical simulation model of splitting grouting, grout inspection coupling environment splitting, diffusion regularity, and comparing with the results of indoor model test and analysis.

\section{The Model Test of Fracture Grouting in Loess}

2.1. Grouting System. The model boxes are made of solid wooden keel and multilayer bakelite plate, with an internal size of $800 \mathrm{~mm} * 800 \mathrm{~mm} * 600 \mathrm{~mm}$ designed. These boxes are dismountable, which allows easy excavation of test samples after grouting [10].

The grouting equipment is a ZBQ-27/1.5 pneumatic grouting pump, with a maximum working pressure of 3.2 $\mathrm{MPa}$ and a maximum displacement of $27 \mathrm{~L} / \mathrm{min}$. The steel pipe with a diameter of $6 \mathrm{~mm}$ is selected as the grouting pipe, and the bottom of the grouting pipe is $400 \mathrm{~mm}$ to the bottom of the box.

The air supply is ensured by FENGBAO $0.12 / 8$ air compressor which provides an output pressure of $0.8 \mathrm{MPa}$. Test system and the grouting pipe are shown in Figure 1.

2.2. Test Materials. The loess used in the test is taken at the construction site from a deep and old stratum. Its physical 


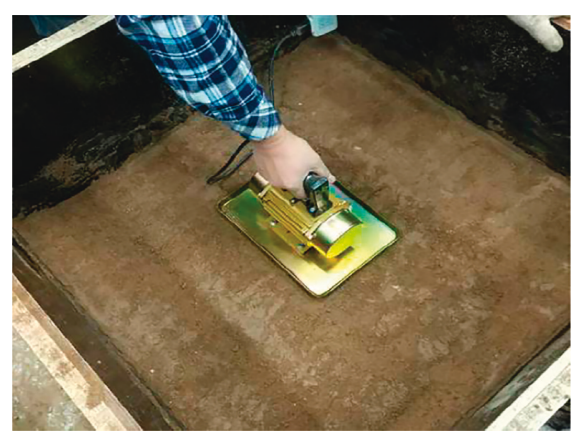

(a)

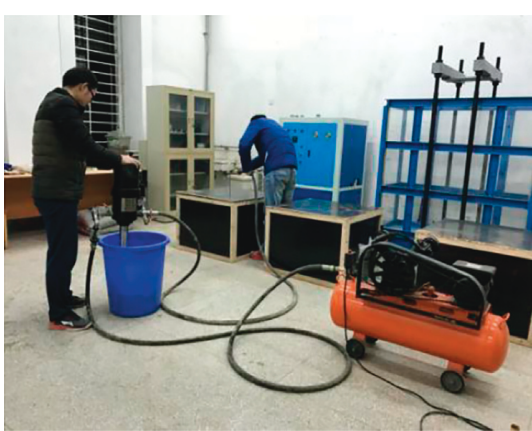

(b)

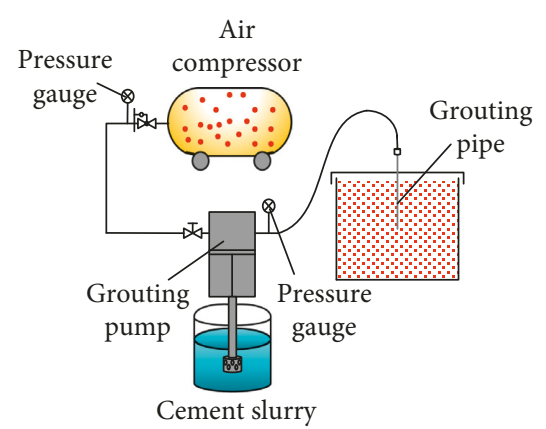

(c)

Figure 1: Test system.

TABle 1: Physical and mechanical indexes of loess.

\begin{tabular}{lcccc}
\hline Soil sample & Water content, $w(\%)$ & Density, $\rho\left(\mathrm{g} \cdot \mathrm{cm}^{-3}\right)$ & Cohesion, $c(\mathrm{KPa})$ & Internal friction angle, $\varphi\left({ }^{\circ}\right)$ \\
\hline Old loess & 17.53 & 1.73 & 31.2 & 25.18 \\
\hline
\end{tabular}

and mechanical indexes are shown in Table 1. During the filling process, the loess was compacted after each $100 \mathrm{~mm}$ of filling to ensure consistency of the mechanical state of the soil mass at the construction site.

The grout used in the test is cement-sodium silicate grout, with water-cement ratio of 0.7 and cement-sodium silicate ratio of $3: 1$ conducted. The cement is Conch ordinary Portland cement P.042.5.

2.3. Numerical Simulation of Particle Flow. In order to effectively reduce the test result discrete and distortion caused by model test conditions, we use the method of numerical simulation to study the mechanism of loess splitting grout.

Particle Flow Code (PFC) is based on a series of discrete processes, using discrete elements to simulate the mechanical behavior of rock soil, which can overcome the limitations of the macroscopic continuity assumption of traditional continuous medium mechanics model, which is more consistent with the actual situation.

Particle Flow Code in 2 Dimensions is based on Newton's second law to describe the interaction between particles in the rock mass and the relative motion of particles, and the discrete element method is used to solve the solution by using a noncontinuous numerical method.

From the microscopic perspective, $\mathrm{PFC}^{2 \mathrm{D}}$ takes the mechanical properties of the material from the physical map to the mathematical field for numerical solution, and uses the abstract particle unit to represent the real granular medium particles. Through the design of the geometric properties of the particle unit, the constitutive model of the particle, the stress balance of the sample, and the boundary conditions of numerical simulation are established, and the macro performance of the numerical calculation corresponds to the mechanical characteristics of the actual process.

In this paper, $\mathrm{PFC}^{2 \mathrm{D}}$ is used by selecting the appropriate particle contact constitutive model and determining the microscopic parameters; the ideal numerical calculation model is established to simulate the loess splitting grouting and further analyze the mechanism of loess splitting grouting.

2.4. Basic Assumptions. It is not possible to directly carry out the macroscopic basic parameter of that model in the numerical calculation of the particle flow, but only by changing the particle size, the geometrical characteristic of the particles, and the interparticle contact characteristic, to establish macroscopic and microscopic relations.

The mechanical properties of the medium depend on the structure and contact characteristics of the particles, and the constitutive character of the model is reflected by the change of state features between the particles, and the destruction and development of the contact between the particles is marked that the mechanical properties of the medium are transformed by linear transformation and are transformed by elastic elastoplastic.

In the numerical calculation of particle flow, the simulation of different properties is realized through the mechanical transfer and interaction of particles. Different contact models have different transfer forces and physical changes between the particles. The constitutive model of the particles determines the constitutive property of the final material.

\section{Determination of Parameters in Numerical Simulation}

3.1. Determination of Loess Grain Parameters. In the particle flow program, the macroscopic mechanical properties of the materials are reflected by setting the constitutive model and the microscopic parameters between the particles.

Based on soil tests, the contact adhesive model simulation of the loess intergranular contact, through a lot of the biaxial particle flow simulation experiment, and the geotechnical test results close to the phase of stress-strain 


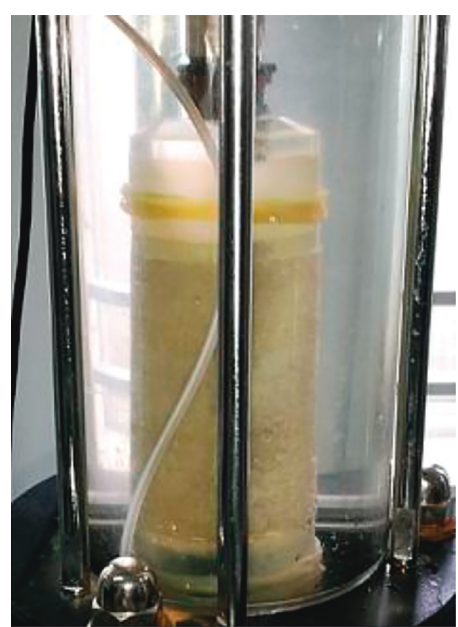

(a)

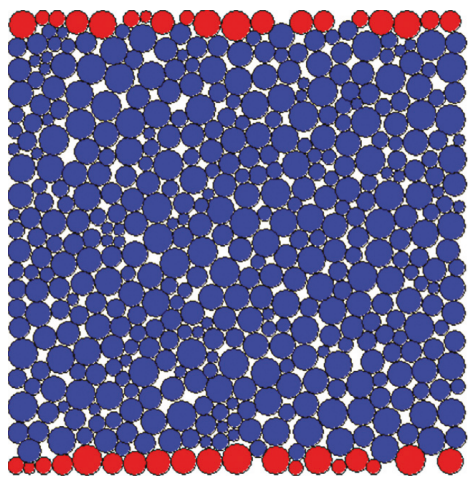

(a)

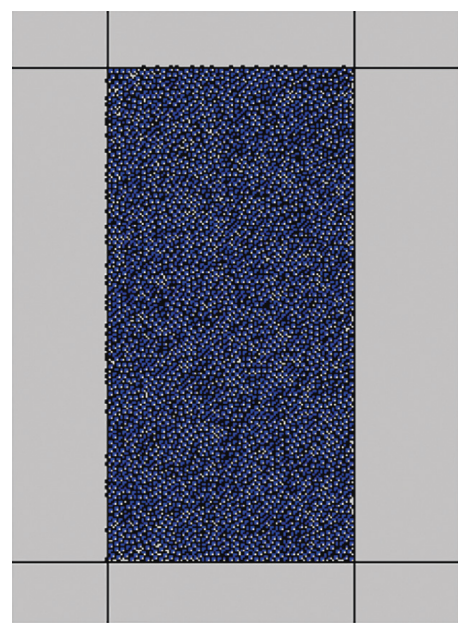

(b)

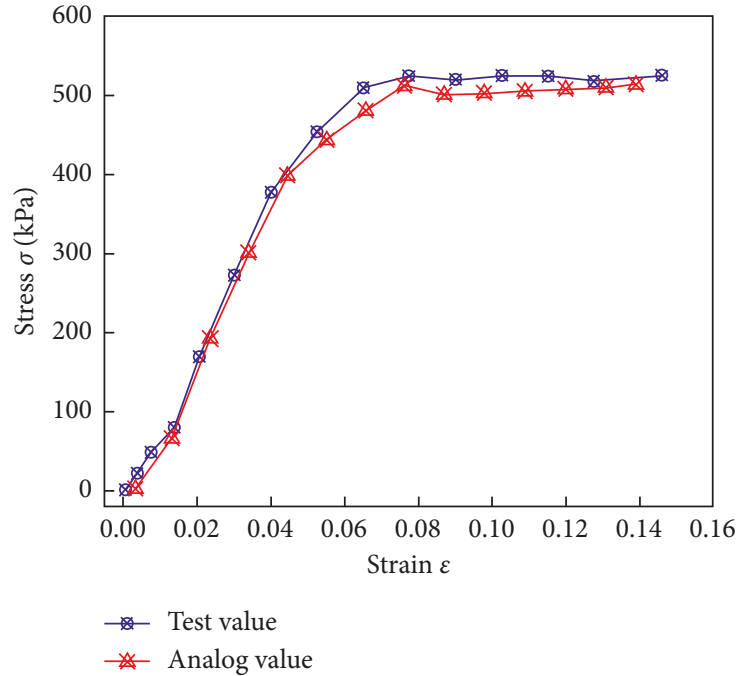

(c)

FIGURE 2: Parameter calibration.

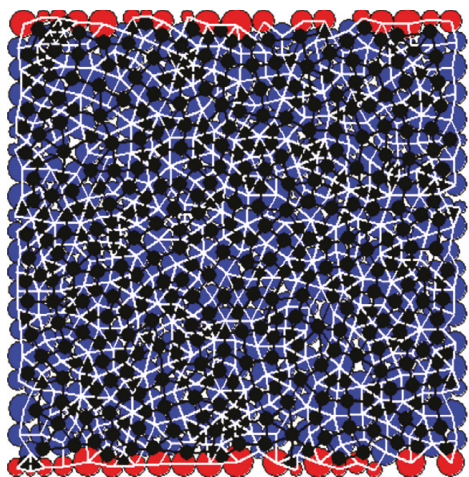

(b)

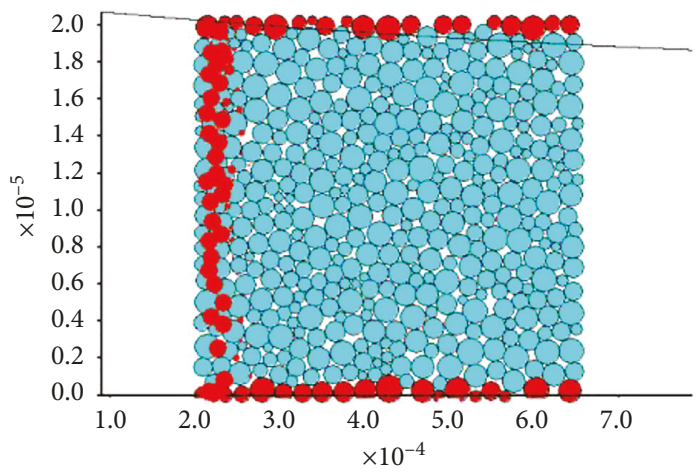

(c)

Figure 3: Osmotic calibration.

TABLE 2: Microparameters of the particle.

\begin{tabular}{|c|c|c|c|c|c|c|}
\hline Particle radius, $R_{\min }$ & $R_{\max } / R_{\min }$ & Contact stiffness, $k_{\mathrm{n}}(\mathrm{N} / \mathrm{m})$ & $K_{\mathrm{n}} / K_{\mathrm{s}}$ & Friction coefficient, $f_{\mathrm{c}}$ & n_bond $(\mathrm{Pa})$ & s_bond $(\mathrm{Pa})$ \\
\hline 0.35 & 1.43 & $2 \times 10^{7}$ & 1 & 0.4 & $2.5 \times 10^{4}$ & $2.5 \times 10^{4}$ \\
\hline
\end{tabular}

curve calibration particles contact mesoscopic parameters such as bond strength, friction coefficient, based on Darcy seepage numerical simulation test for calibration of the coefficient of permeability of loess, and got to be able to reflect the loess macro mechanical properties of the numerical model of the material. The process of parameter calibration is shown in Figures 2 and 3.

In the calculation, we use four walls of the wall to simulate the boundaries of the earth model and the loading process of the particles. The upper and lower "walls" simulate the loading tray and the left and right "walls," through the servo control program, apply the fixed confining pressure and control the movement speed Table 2 .

It was found that stress-strain curve of the biaxial simulation fits well with the result of indoor geotechnical test at $100 \mathrm{kPa}$ by comparing the fit of stress-strain curve under different conditions. The parameters can be applied to the microscopic numerical simulation of loess fracturing grouting [11-13].

3.2. Determination of Permeability Coefficient. The dimension of the numerical calculation model is $4 \mathrm{~m} \times 4 \mathrm{~m}$, which is filled with 2050 particles to simulate the actual formation structure. In $\mathrm{PFC}^{2 \mathrm{D}}$, the calibration and value of loess permeability coefficient is carried out by means of analog Darcy osmosis test. Four "wall" units are generated in the boundary position, and the "ball" units of different sizes are generated at random locations in the internal space. The radius is subject to the uniform distribution within the interval of $0.35 \mathrm{~mm}$ to $0.5 \mathrm{~mm}$, which can reflect the inhomogeneity of soil samples in nature. 
TABLE 3: Microparameters of fluid.

\begin{tabular}{|c|c|c|c|c|c|c|c|}
\hline $\begin{array}{l}\text { Penetration } \\
\text { coefficient }\end{array}$ & $\begin{array}{c}\text { Head } \\
\text { pressure } \\
(\mathrm{Pa})\end{array}$ & $\begin{array}{l}\text { Volume of } \\
\text { domain, } V_{\mathrm{d}} \\
\left(\mathrm{mm}^{3}\right)\end{array}$ & $\begin{array}{l}\text { Number of pipes in } \\
\text { a domain }(\mathrm{N})\end{array}$ & $\begin{array}{l}\text { Conduction } \\
\text { coefficient, } K \\
\left(\mathrm{~m} \cdot \mathrm{s}^{-1}\right)\end{array}$ & $\begin{array}{c}\text { Fluid volume } \\
\text { modulus, } \\
K_{\mathrm{d}}(\mathrm{kPa})\end{array}$ & $\begin{array}{l}\text { The diameter of } \\
\text { pipe, } a(\mathrm{~mm})\end{array}$ & $\begin{array}{l}\text { Time, } \\
\Delta t(\mathrm{~s})\end{array}$ \\
\hline $1.31 \times 10^{-5}$ & $1.0 \times 10^{6}$ & 1.0 & 2 & $1.0 \times 10^{-5}$ & $1.0 \times 10^{6}$ & 1.0 & $1.0 \times 10^{-2}$ \\
\hline
\end{tabular}

TABLE 4: Initial stage test and simulation process.

\begin{tabular}{llcl}
\hline Period & Foundation stone & Grout ball & First fracture
\end{tabular}

Diffusion process
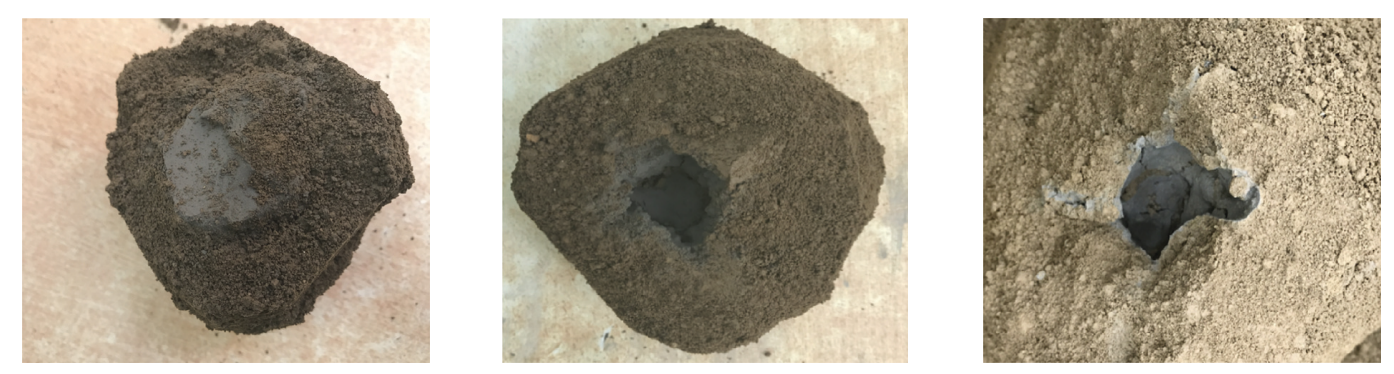

Simulation process
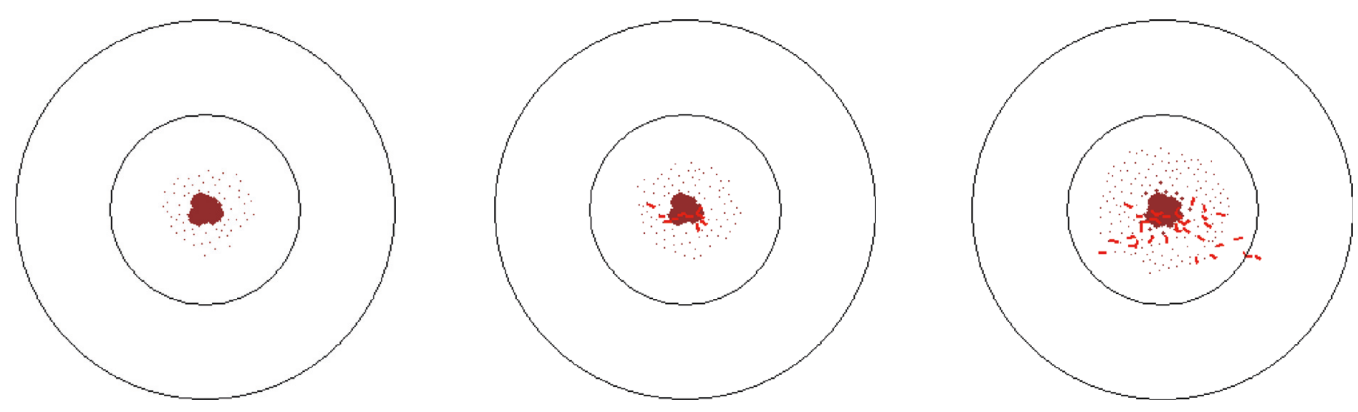

Analysis of maximum shear stress
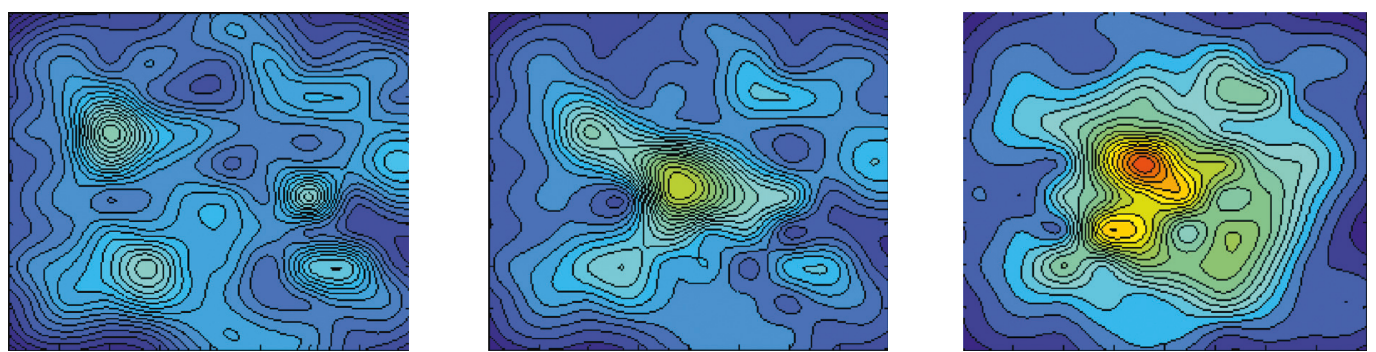

After the system is balanced, the particles of the upper and lower boundaries are fixed, and the soil model is formed, as shown in Figure 3.

The "domain" and "pipeline" are generated between the particles, and the network is formed to simulate the movement of the fluid and the interaction with the particles. Figure 3 is a schematic diagram of the fluid domain and piping in the model, and the blue ball indicates soil particles. Black balls is the "domain", that can hold liquid. Black channel between the "domain" to "pipe," can pass "domain" on both ends of the pipe in the liquid, and the pipeline on both sides of the particles. There is no "pipe" in the upper and lower boundaries, which is defined as impermeable layer.

These parameters are adjusted in the Darcy penetration test. When the permeability coefficient is measured and the expected permeability coefficient is obtained, the calibration of the permeability coefficient is completed, and the numerical simulation can be carried out directly Table 3 .

3.3. Analysis of Fracture Grouting in Loess. The basic parameters of the loess used for the test are shown in Table 1. During the test, the grouting pressure was controlled from 0 to $0.4 \mathrm{MPa}$, and the grouting time was controlled within $2 \mathrm{~s}$. There are six stages in process of grout fractures in loess.

3.3.1. Compaction Stage $(P<0.1 M p a, T>0 s)$. After the start of grouting, the grouting pressure had not yet reached the point of fracturing. When the grout was injected into the 
TABLE 5: Fracture stage test and simulation process.

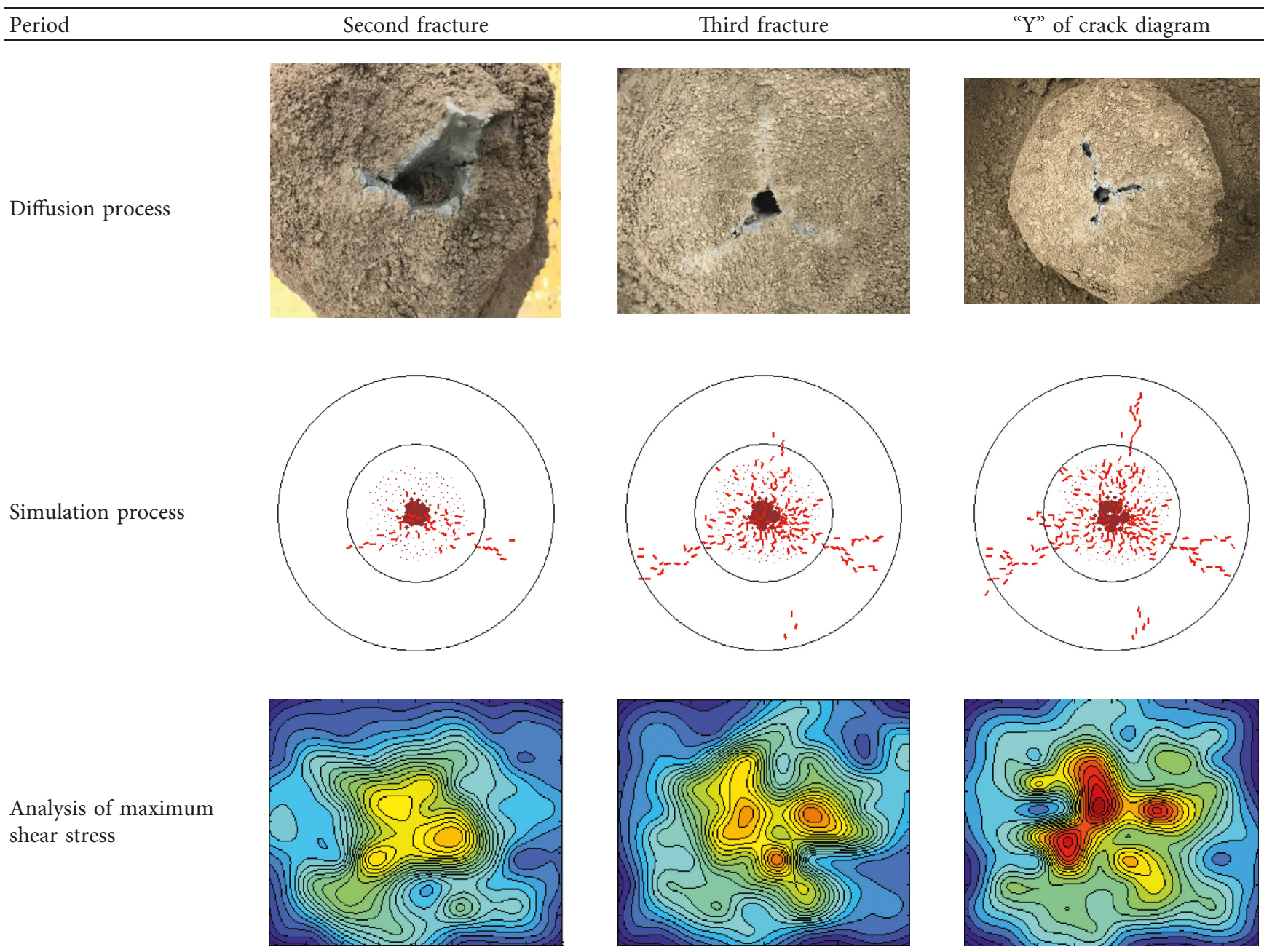

soil mass, the soil mass was impacted and compacted, and a "foundation stone" was gradually formed [14].

\subsubsection{Expansion and Compaction Stage $(P<0.2 M P a, T>0 s)$.} With the increase of the grouting pressure, the grout continued to accumulate, driving the "foundation stone" closer to the grouting pipe nozzle. As the grout could not break through the "foundation stone," which splashed and expanded the soil mass around, grout balls formed gradually.

The formation of grout balls produced a compaction effect on the soil, leading to the plastic deformation of the soil mass. The energy in the plastic deformation was stored in the form of plastic strain energy [5]. As the pressure of the grout increases, the soil around the grouting hole is densely packed, and stress concentration phenomenon occurs, and the maximum shear stress of local soil particles is greater than the initial setting value of the tangential cohesive strength of the particles, which causes the occurrence of the cohesive bond between the particles and the occurrence of microcrack, but the direction of the cleavage crack cannot be predicted from macroscopically. The test and simulation of grouting are shown in Tables 4 and 5.

3.3.3. First Fracturing Stage $\left(P=P^{1}=0.25 M P a, T=0.5 s\right)$. With the increase of the grouting pressure, the plastic strain energy in the soil mass and the energy accumulated in the grout to split the soil mass, as well as the compressive force and effective stress of the grout on the soil mass, were also increasing during the process [5]. When the grouting pressure reached $0.25 \mathrm{MPa}$, the soil yielded and failed under the effect of compressive force. The grout was pumped in from the weakest plane of the soil mass. This led to the appearance of the new fracture, which was later filled by the grout.

3.3.4. Second Fracturing Stage $\left(P=P^{2}=0.3 M P a, T=0.835 s\right)$. When the first grout fracture was filled, the soil mass was compacted and subject to plastic deformation, allowing the grout balls to form a closed loop again. During the process, the weakest plane of the soil mass is constantly changed [5]. The formation of the first grout fracture made the soil 


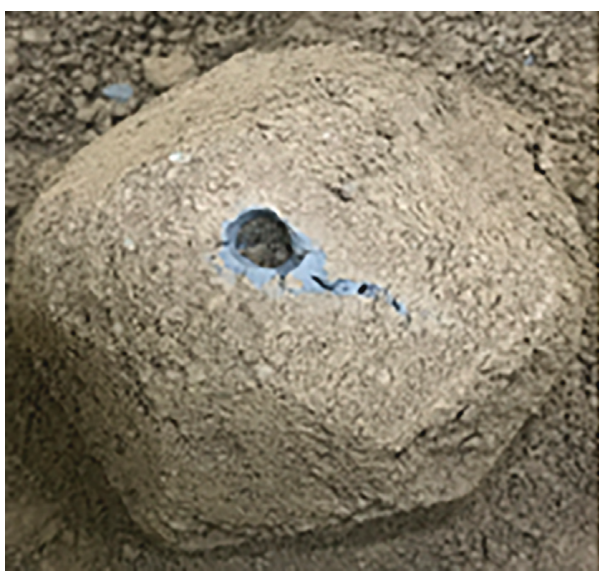

(a)

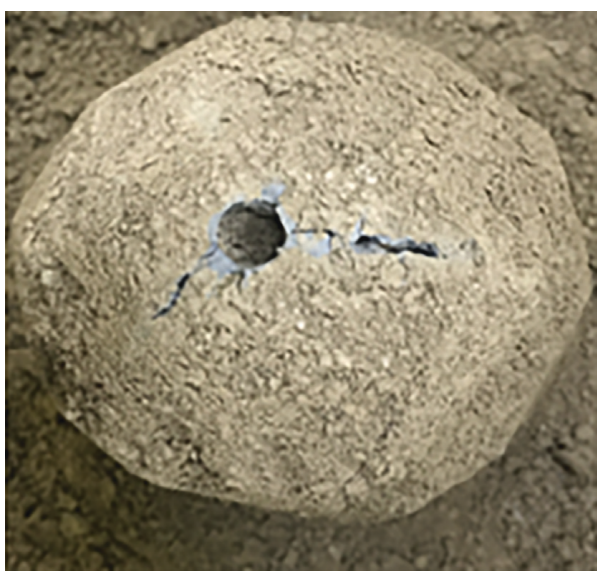

(b)

FIgURE 4: Sectional view.

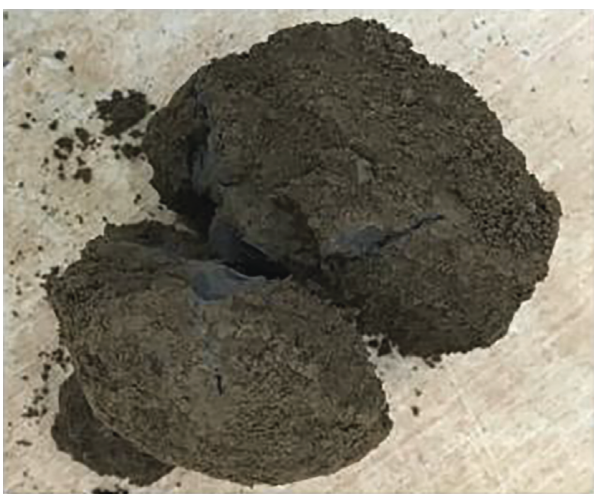

(a)

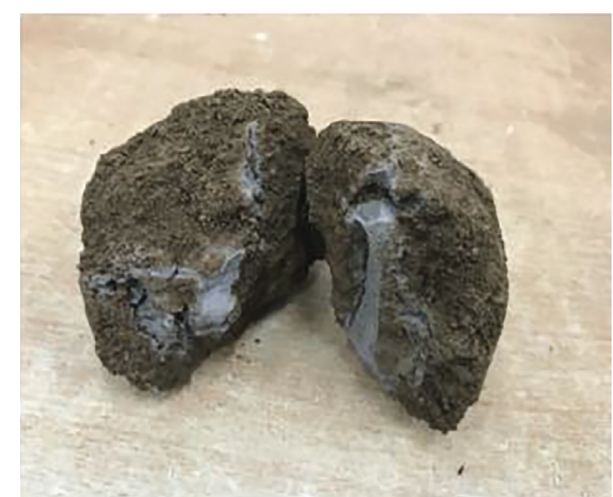

(b)

FiguRE 5: Vertical view.

compacted. When the grouting pressure reached the second peak value of $0.3 \mathrm{MPa}$, the energy accumulated in the grout might even split the soil mass and form a diffusion path at $120^{\circ}$ to the first grout fracture.

Due to the development of cracks and the rise of pressure, the main stress direction in the stratum starts to transform, and the horizontal direction of the main stress is converted into the passive earth pressure state. At this point, it is necessary to have more grouting pressure to widen the crack in the soil, and a second peak of pressure occurs. Because at this time, the level of stress is greater than the vertical, and the formation starts to go up to the fracture and the second crack.

3.3.5. Third Fracturing Stage $\left(P=P^{3}=0.35 M P a, T=1.2 s\right)$. When the grouting pressure reached the third peak value of $0.35 \mathrm{MPa}$, the energy accumulated in the grout might even split the soil mass. In this case, a third grout diffusion path was generated, which was at approximately $120^{\circ}$ to the other two grout fractures. At this point, all three fractures of the fracture grouting in loess were formed.
3.3.6. Common Development Stage ( $P=0.35 \mathrm{MPa}, \mathrm{T}>1.2 \mathrm{~s})$. The samples were cut horizontally layer by layer from top to bottom. The three grout fractures appeared in turn, at approximately $120^{\circ}$ to one another.

3.4. The Formation of " $Y$ " Type Diffusion Form. This phenomenon shows that the grout splits the soil mass for three times, resulting three grout fractures forming at different heights and with different lengths; from the longitudinal section of the sample, it is found that the grout fractures are generated and filled from bottom. They spread away from near the grouting hole and become thinner and thinner. In addition, infiltration is observed at the end of the grout fractures due to the pressure in the diffusion process. The sectional view and the vertical view are shown in Figures 4 and 5 .

\section{Conclusions}

(1) With the increase of the grouting pressure, the grout changes with the pressure in six stages, foundation, expansion and fracturing, and diffusion, and 
ultimately splits into three grout fractures (at approximately $120^{\circ}$ to each other).

(2) Through the use of $\mathrm{PFC}^{2 \mathrm{D}}$ particles flow numerical simulation program, under the environment of the coupling dynamic process of the loess fracturing grouting mesoscopic simulation, analyzing different grouting pressure and grout diffusion on at the same time, the rule of the slurry is obtained in the loess splitting " $\mathrm{Y}$ " in the space geometry.

(3) The fracture is caused by the maximum shear stress of the local soil particles larger than the initial set value of the grain tangential bond strength, which causes the fracture of the bond between particles, and explains the mechanical mechanism of the formation of the split crack.

\section{Conflicts of Interest}

The authors declare that they have no conflicts of interest.

\section{References}

[1] Y.-W. Guo and S.-H. He, "Theoretical study of plane equivalent elastic model of composite soils with fracturing grouting," Rock and Soil Mechanics, vol. 36, no. 8, pp. 21932200, 2015.

[2] T.-F. Xie, W.-B. Xie, S.-G. Jing et al., "A mathematical model for fracture grouting in underground engineering," Modern Tunnelling Technology, vol. 52, no. 2, pp. 104-109, 2015.

[3] Q.-S. Zhang, L.-Z. Zhang, R.-T. Liu et al., "Split grouting theory based on slurry-soil coupling effects," Chinese Journal of Geotechnical Engineering, vol. 38, no. 2, pp. 323-330, 2016.

[4] M.-L. Huang, X.-M. Guan, Q.-F. Lü et al., "Mechanism analysis of induced fracture grouting based on elasticity," Rock and Soil Mechanics, vol. 34, no. 7, pp. 2059-2064, 2013.

[5] P. Li, Q.-S. Zhang, X. Zhang et al., "Analysis of fracture grouting mechanism based on model test," Rock and Soil Mechanics, vol. 35, no. 11, pp. 3221-3230, 2014.

[6] Z.-M. Zhang, J. Zou, J.-Y. Jia et al., "Laboratory tests on compaction grouting and fracture grouting of clay," Chinese Journal of Geotechnical Engineering, vol. 31, no. 12, pp. 1818-1824, 2009.

[7] Q.-C. Wang and R.-L. Zhang, "Experimental research on the trend of slurry about fracturing grouting and soil displacement in different pressure," Journal of the China Railway Society, vol. 33, no. 12, pp. 107-111, 2011.

[8] F. Sun, D.-L. Zhang, T.-L. Chen et al., "Meso-mechanical simulation of fracture grouting in soil S," Chinese Journal of Rock Mechanics and Engineering, vol. 32, no. 3, pp. 474-480, 2010.

[9] P.-F. Qin, "Mesomechanics particle flow numerical simulation research on sandy soil bio-grouting," Rock and Soil Mechanics, vol. 37, no. 1, pp. 603-608, 2016.

[10] Z. Zhang, X. Fang, Z. Shao, and Y. Xu, "Experiment of fracture grouting mechanism in loess and its application," Electronic Journal of Geotechnical Engineering, vol. 22, no. 11, pp. 4419-4436, 2015.

[11] X.-H. Xue, J. Zhang, Z.-M. Su et al., "Grouting prereinforcement mechanism and effect evaluation in waterrich loess tunnel," Journal of Chongqing Jiaotong University, vol. 34, no. 4, pp. 34-38, 2015.
[12] S.-C. Wu, Studies and Application of Ground Treatment by Composite Anchor Pile with Pressure Grouting, Beijing University of Science and Technology, Beijing, China, 2004.

[13] F.-J. Song, The Method of Numerical Simulation of Deep Hole Grouting Reinforcement Effect in Subway Engineering, Beijing University of Civil Engineering and Architecture, Beijing, China, 2015.

[14] J.-F. Zou, L. Li, X.-L. Yang et al., "Energy dissipation analysis for crack grouting," China Railway Society, vol. 27, no. 2, pp. $52-55,2006$. 


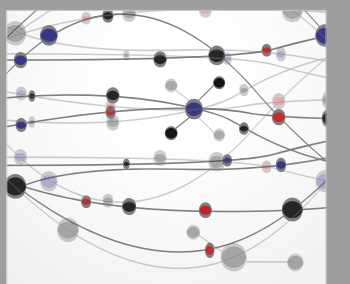

The Scientific World Journal
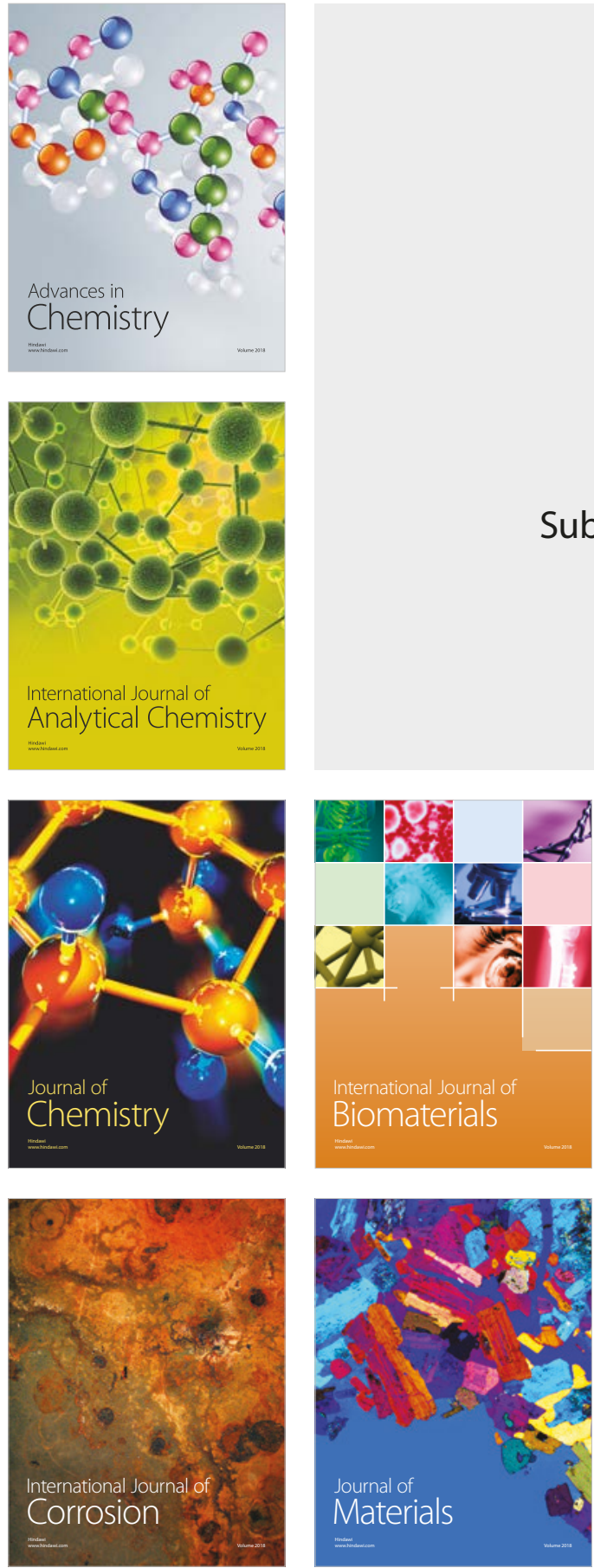

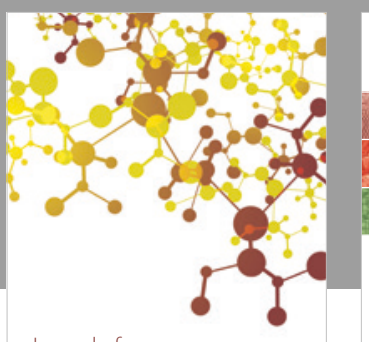

Journal of

Applied Chemistry
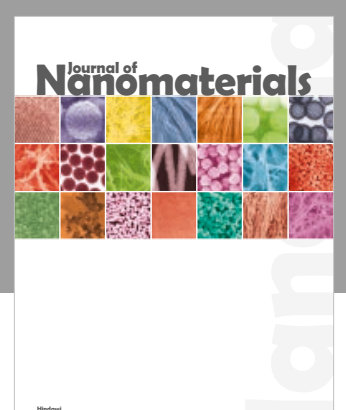

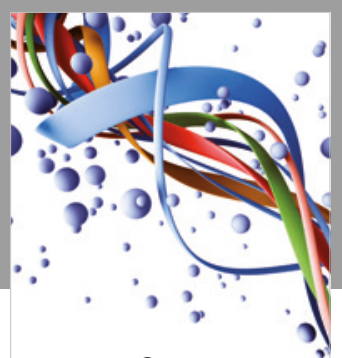

Scientifica

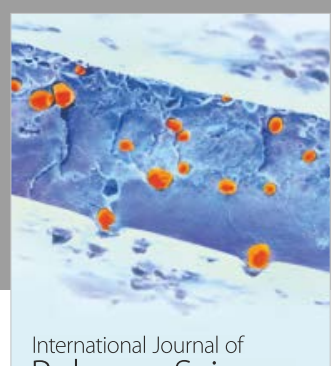

Polymer Science

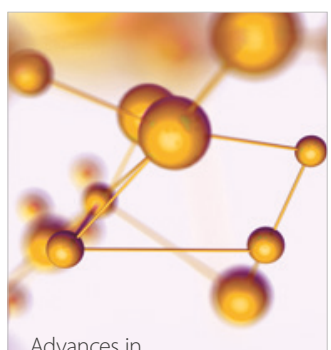

Physical Chemistry
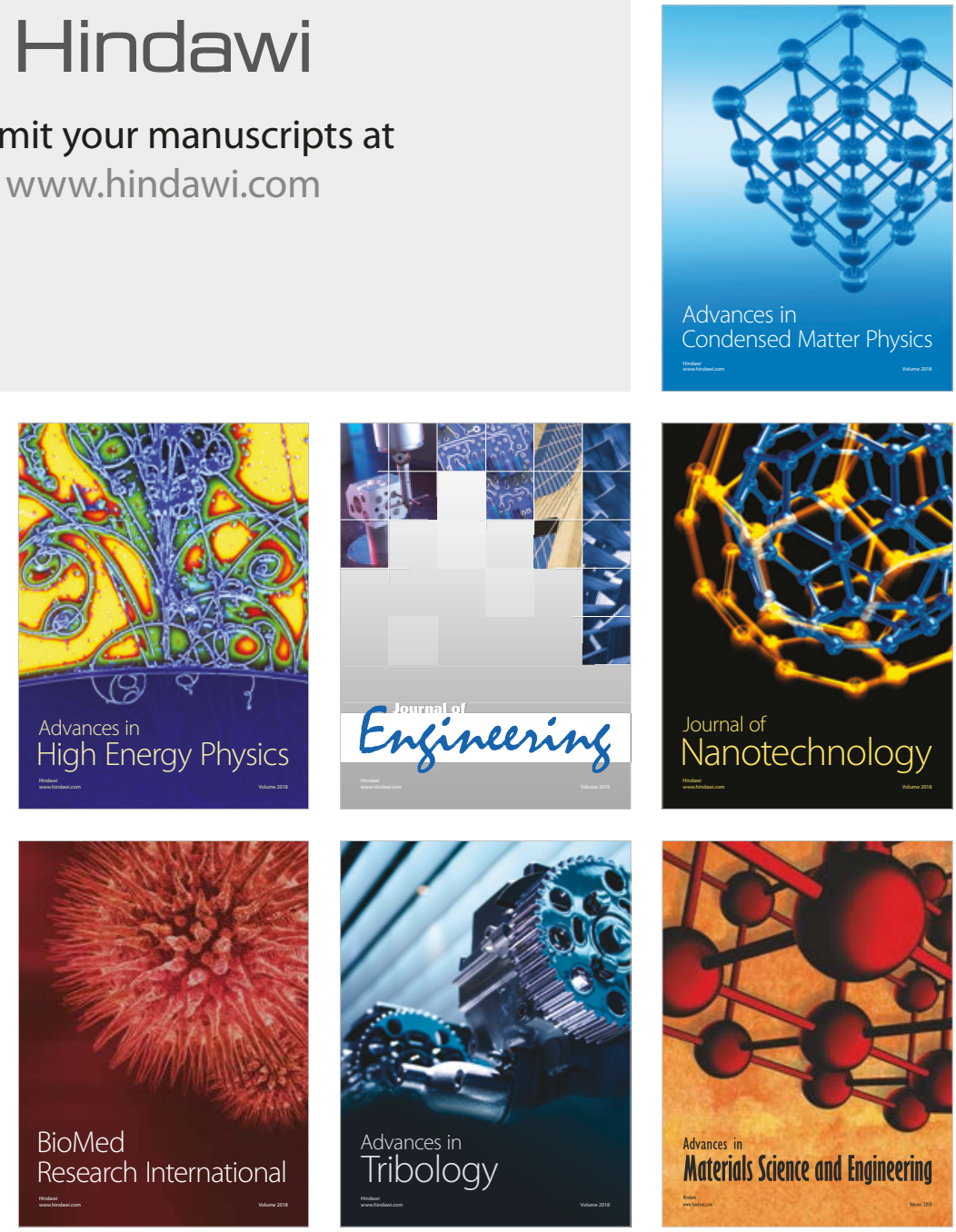